\title{
Intra-Channel Interference Avoidance with the OGFDM Boosts Channel Capacity of Future Wireless Mobile Communication
}

\author{
Mohammad R. Kadhum, Triantafyllos Kanakis, and Robin Crockett \\ Faculty of Arts, Science \& Technology, University of Northampton, Northampton, \\ U.K. \\ mohammad.kadhum@northampton.ac.uk
}

\begin{abstract}
The Orthogonal Generalized Frequency Division Multiplexing (OGFDM) with Intra-Channel Interference Avoidance (ICIA) approach is, for the first time, proposed, explored and evaluated. Since the interference manipulation currently represents a hot topic for wireless mobile communication, the conventional approach of mitigating the interference is no longer acceptable. As a result, a novel method for addressing the interference between adjacent filtered sub-carriers (inphase/out-phase) is comprehensively investigated herein. The proposed approach utilises the oversampling factor to effectively avoid interference and improve the quality of service of affected filters under bad transmission states. Thus, this supportive method which is essentially aware of propagation conditions is employed for removing the roll-off $(\alpha)$ impact yet improving the level of Bandwidth (BW) efficiency for applied filters of the OGFDM waveform. Besides, in terms of the system performance, the trade-off relation between the channel capacity and the key Hilbert filter parameter is theoretically and practically discussed. This requires investigation of the influence of $\alpha$ factor on the maximum achieved bitrate at the acceptable limit of the Bit Error Rate (BER). A MATLAB simulation was introduced to test the performance characteristics of the proposed system in the physical layer (PHY) of an electrical back-to-back wireless transmission system.
\end{abstract}

Keywords: Orthogonal Generalized Frequency Division Multiplexing (OGFDM); Intra-Channel Interference Avoidance (ICIA); Bit Error Rate (BER); Hilbert filter; roll-off factor; oversampling factor; wireless mobile communication; channel capacity; physical layer (PHY).

\section{Introduction}

The rapid growth in mobile networks and big limitation of the current mobile waveform have pushed the cellular networks developers to explore new transmission technologies that can improve the channel capacity and deliver a more reliable service to the user in comparison to the present $4 \mathrm{G}$ standard [1]. Thus, due to the key drawbacks of the Orthogonal Frequency Division Multiplexing (OFDM) 
[2], new candidate waveforms like the Filter Bank Multi-Carrier (FBMC) [3], the Universal Filter Multi-Carrier (UFMC) [4], the Filtered OFDM (F-OFDM) [5], and the Generalized Frequency Division Multiplexing (GFDM) [6] are proposed to the future scenarios of the next mobile generation (5G).

Moreover, lately, a new candidate waveform termed as Orthogonal Generalized Frequency Division Multiplexing (OGFDM) [7] is presented with extra supportive facilities to the $5 \mathrm{G}$ mobile applications. Hence, the recently introduced waveform can achieve a dual channel capacity yet keeping the same level of error relative to the most candidate waveform GFDM.

It's worth noting that the need for a higher channel capacity pushes the networks operators to upgrade the usage of the Bandwidth (BW) by increasing the number of employed frequency sub-carriers. On the other hand, the increased number of frequency sub-carriers can raise the level of interference, particularly, with bad transmission conditions [8].

Due to the predicted scenarios of the future generation of mobile, particularly, higher channel capacity $(\mathrm{Gb} / \mathrm{s})$, the conventional management for the induced interference of wireless mobile networks is no longer applicable [9]. The main reason beyond this that the traditional approach can mitigate the interference with wasting the BW efficiency of affected frequency sub-carriers [10]. Hence, depending on the traditional approach, the delivered amounts of channel capacity are decreased severely after mitigating the affected sub-carriers.

As a result, the new candidate waveforms, especially, the best candidate one "OGFDM" needs to a modern manipulation for the interference makes it able to cope with the future market demands of mobile communications [11].

In this paper, a new approach called Intra-Channel Interference Avoidance (ICIA) for removing the interference between the adjacent filtered sub-carriers of the OGFDM yet improving the efficiency of the BW is investigated.

The proposed scheme employs the oversampling operation to efficiently avoid any probable bad behaviour of the $\alpha$ factor for utilised filters. Thus, by controlling the oversampling factor, new guard intervals are generated between the filtered sub-carriers to accommodate optimally the expanded cases of the $\alpha$ factor.

In addition, the organized distances between frequency centres $\left(f_{c} s\right)$ of the employed filters are fixed optimally. Hence, assure, primarily, that all Nyquist intervals of sub-carriers and their filters are mostly secured and followed a similar allocation procedure for variant $f_{c} s[12]$.

Also, under a standard level of the transmission circumstances (good signal power), the transmitted data are distributed and carried by different $f_{c} s$, then to be received sequentially by the counterparts of them [13].

Furthermore, the introduced approach is basically achieved in the physical layer (PHY), where the system can manage the assigned value of the oversampling factor in accordance with the $\alpha$ parameter of the filter.

Regarding the changeable conditions of the transmission, the trade-off relationship between the overall channel capacity and the $\alpha$ factor of filter is investigated to identify the optimal guidelines of filter design under various transmission 
status. Hence, explore the system performance of the transmitted signal which may suffer from power loss during its travel to the receiver to ensure a secured transmission for all situations.

The level of power attenuation is varied to optimal, acceptable and severe due to the conditions of transmission. As such, the possibility of interference occurrence between rolled-off filters is increased if the level of filter expansion is enlarged due to the decreased average of transceiver power [14].

The rest of the paper is structured as follows: Section 2 discusses theoretically the main advantages and disadvantages of the proposed system in an electrical back-to-back wireless mobile system. Section 3 evaluates the transmission performance in terms of the channel capacity and Bit Error Rate (BER) of the OGFDM transceiver utilising a MATLAB simulation code. Section 4 summarises the key outlines of the paper.

\section{System Model}

As is shown in Fig. 1, in the transmission operation, firstly, each sub-carrier in the OGFDM system is digitally encoded. Then, the complex numbers are produced by employing one of the most popular modulation formats, like BPSK, 16 QAM, 128 QAM, etc.

After that, in the ICIA part, where important manipulations that directly related to the oversampling operation are achieved, each modulated sub-carrier is up-sampled dynamically either by $K$ or $2 K$ factor depending on the applied system (conventional/dual). Hence, insert a set of zeros, equivalents to the utilised factor of oversampling, between every two adjacent samples of original data.

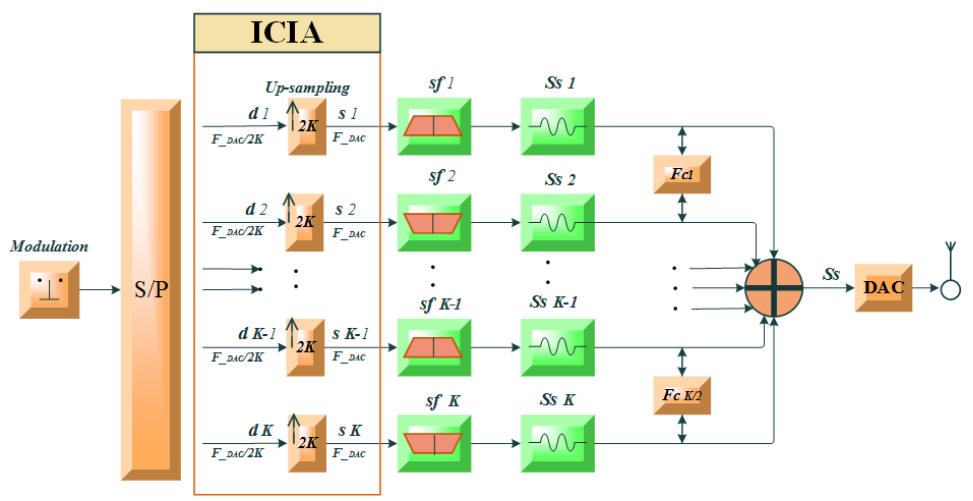

Fig. 1. Transmitter side of the OGFDM with ICIA.

Subsequently, the up-sampled sub-carriers are passed through Hilbert shaping filters. As a result, every two adjacent sub-carriers are filtered orthogonally and assigned to a similar $f c$. Hence, every single $f c$, can be shared between 
two different sub-carriers simultaneously. Thus, accommodate an extra number of filtered sub-carriers (double) for the same BW.

In addition, to ensure optimum performance for the filtering process, key shaping filter parameters like the coefficients set are adjusted precisely in the PHY.

Having been filtered digitally, different convoluted sub-carriers from various $f_{c} s$ are then combined utilising the adder in the digital domain. The out coming sequence of data is then pushed through a digital-to-analog converter (DAC) generating the analog signal. Finally, the electrical analog signal is propagated through the antenna.

As is clear in Fig. 2, in the reception operation, the received analog electrical signal is firstly digitized employing an analog-to-digital converter (ADC). Thereafter, the stream of data corresponding to each sub-carrier in the OGFDM system are de-multiplexed using equivalent matching filters.

To achieve a successful filtering operation for all employed matching filters, the matching coefficients are similarly adjusted to their shaping counterparts.

After matching and de-multiplexing process of the received sub-carriers via the digital filters, the up-sampled sub-carriers are then down-sampled in the ICIA part, where, oversampling operation is manipulated by removing all previously embedded zero samples. Ultimately, during the de-modulation process, the original data is retrieved utilising a previously decided modulation format.

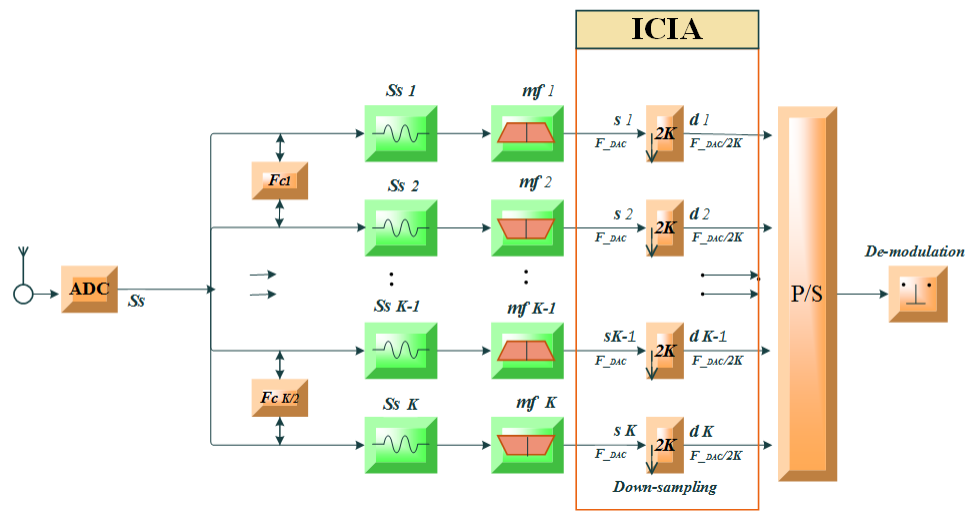

Fig. 2. Receiver side of the OGFDM with ICIA.

Mathematically speaking, the key required operations of the proposed transceiver system are demonstrated. Thus, on the transmitter side, the impulse responses of the $h^{t h}$ shaping pairs of Hilbert filters are written as follows [15]:

$$
\begin{aligned}
& s f_{h}^{A}(t)=g(t) \cos \left(2 \pi f c_{h} t\right) \\
& s f_{h}^{B}(t)=g(t) \sin \left(2 \pi f c_{h} t\right)
\end{aligned}
$$


where $f c_{h}$ represents the centre frequency of the $h^{\text {th }}$ Hilbert filters pairs, and $g(t)$ is the baseband pulse which is expressed as follows [15]:

$$
g(t)=\frac{\sin [\pi(1-\alpha) \gamma]+4 \alpha \gamma \cos [\pi(1+\alpha) \gamma]}{\pi \gamma\left[1-(4 \alpha \gamma)^{2}\right]}
$$

where $\gamma=t / \Delta t, \alpha$ parameter supervises the excess of filter BW and $\Delta t$ is corresponding to the sampling interval before the oversampling. In addition, the in-phase and quadrature-phase of the Hilbert filter pair components are denoted by the superscripts $A$ and $B$ respectively. Its worth noting that each of those components is utilised independently to convey a sub-carrier. Moreover, on the receiver side, a version of impulse responses of matched filters for the corresponding shaping filters of Hilbert-pair are written as follows [15]:

$$
\begin{aligned}
& m f_{h}^{A}(t)=s f_{h}^{A}(-t) \\
& m f_{h}^{B}(t)=s f_{h}^{B}(-t)
\end{aligned}
$$

To recover the signal at the receiver side, convolution applied between shaping and matching filters achieves the following [15]:

$$
s f_{j}^{C}(t) \otimes m f_{j}^{D}(t)=\left\{\begin{array}{ll}
\delta\left(t-t_{0}\right) & \text { if } \mathrm{C}=\mathrm{D} \text { and } \mathrm{j}=\mathrm{i} \\
0 & \text { if } \mathrm{C} \neq \mathrm{D} \text { or } \mathrm{j} \neq \mathrm{i}
\end{array}\right\}
$$

where $t_{0}$ represents the probable delay time, the superscripts $C$ and $D$ indicate either in-phase or quadrature-phase level, and the subscripts $i$ and $j$ refer to the position of the $f c$.

To support the normal case of employing $K$ filtered sub-carriers in the proposed system, the value of the oversampling factor $(O V)$ is set to be equivalent to the total number of applied sub-carriers $(O V=K)$. Furthermore, considering that the sampling speeds of the ADC and the DAC are identical, the decision of central frequency allocation for each pair of Hilbert filters is determined.

In this context, frequency responses of Hilbert filters are optimally distributed due to the perfect selection for each $f c$ utilised in the spectral region of the obtainable DAC/ADC. Thus, filters accommodation process is performed orthogonally and sequentially considering both phases of each $f c$.

The key measurement value for representing the BW excess of each filtered sub-carrier is the $\alpha$ factor. Hence, whenever the $\alpha$ factor is increased, that means, the drop in the amplitude or power is raised and the level of interference between adjacent filters is growing.

To explain more about the introduced process, it is seen from Fig. 3 that each pair of orthogonal filters works on two copies of oversampled sub-carrier. For example, for each specific $f c$ of filters' pair, the first copy of the first oversampled sub-carrier is taken by the in-phase filter (cosine filter) and the second one of the second oversampled sub-carrier is taken by the out of phase filter (sine filter) simultaneously. Consequently, odd and even sub-carriers are orthogonally filtered by the in-phase and out-phase filters respectively. 


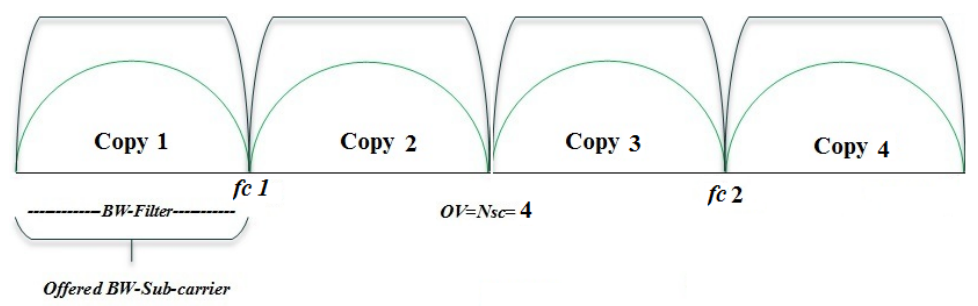

Fig. 3. Normal oversampling for 4 filtered sub-carriers.

The BW size of utilised filters $F_{B W}$ depends on two major parameters. Firstly, the offered sampling rate of the employed sub-carrier $S u b_{S}$ and secondly, the value of BW excess ( $\alpha$ factor) of applied filters. The relation between the allocated filter BW and these two key factors is expressed as follows [16]:

$$
F_{B W}=S u b_{S} *(1+\alpha)
$$

where $1>=\alpha>=0$.

Regarding the first significant factor, the BW of each filter varies according to the size of an individual copy in an oversampled sub-carrier. Such a variation is due to change in the configuration of oversampling operation, hence, size of the filter BW decreased whenever the value of the $O V$ is increased and vice-versa.

To explain more about this, it is clear in Fig. 4 that if the specified BW for each convoluted sub-carrier is less than originally afford BW by the system, the aggregated channel capacity is affected badly, particularly, utilising a similar $f c$ for different oversampling cases. Hence, compared to the optimally obtained sub-carrier $\mathrm{BW},\left(B W=F_{D A C} / O V\right)$, where the $O V$ is equivalent to the number of applied sub-carriers $K$, the outcome $\mathrm{BW}$ for each filtered sub-carrier after applying the dual value of the $O V$ will be reduced by a half, $\left(B W=F_{D A C} / O V\right.$, where, $\left.O V=2 K\right)$. As a result, the overall channel capacity is decreased according to Shannon theorem as follows [16]:

$$
\operatorname{Cap}_{I C I A}=(B W / 2) * \log _{2}(1+S N R)
$$

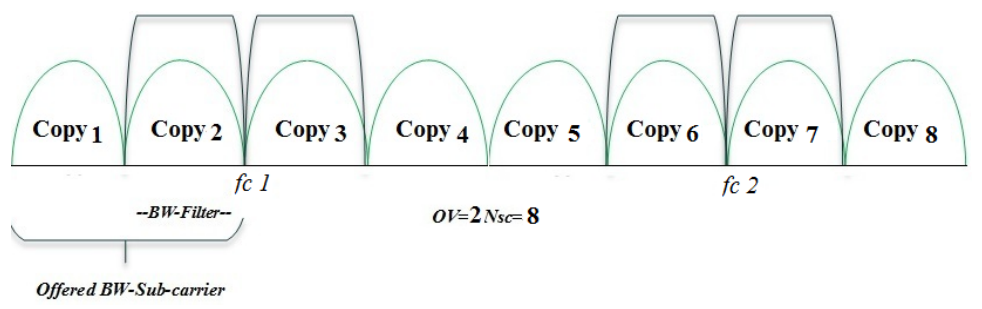

Fig. 4. Dual oversampling for 4 filtered sub-carriers. 
Regarding the second noteworthy factor, if $\alpha$ factor has a zero value, the allocated BW of each utilised filter is equal to the offered sampling rate of each employed sub-carrier. Thus, the produced brick wall filter for both in-phase and out-phase levels has no interference with its adjacent filters which is considered as an ideal case [17].

On the other hand, setting $\alpha$ value to its maximum $(\alpha=1)$ leads to double the occupied size of each applied filter. Hence, increase the interference between them, especially, with normal oversampling state [18].

From the time-domain perspective, oversampling operation does not affect transmission time specified for each OGFDM iteration absolutely. Hence, multiply the number of samples by $X$ does not increase the overall delay time for each symbol since the smallest duration between any two adjacent samples will be divided by $X[19]$.

For example, the calculated time $T_{1}$ for $N$ transmitted samples of an individual OGFDM iteration before oversampling process is, $T_{1}=N * d t$, where $d t$ represents the closest duration between any two adjacent samples. After oversampling the sub-carrier by $X$, the counted time $T_{2}$ for transmitting $N X$ samples of an individual OGFDM symbol is, $T_{2}=N X * d t / X$, which is consequently equivalent to $T_{1}$.

To ensure that the convolution between an oversampled sub-carrier and the applied filter is achieved perfectly, the frequencies for both filter and sub-carrier must be identical [20]. Hence, the frequency for each adopted sample of an individual sub-carrier $\left(f_{i}\right)$ is calculated accurately and then to be considered as a first input to the convolution process. The second input is the calculated frequency for the corresponding selected sample of the applied filter $\left(f_{j}\right)$. If frequencies for samples of both oversampled sub-carrier and utilised filter are similar, $\left(f_{i}=f_{j}\right)$, the convolution is achieved successfully.

Occasionally, the variance between any two adjacent taps in the applied filter is rather large, thus, the most common solution to come up with this issue is by increasing the number of utilised taps of the filter. The calculated frequency for a new tap achieved between two adjacent frequencies of the utilised filter $\left(f_{j}\right.$, $f_{j+1}$ ) will be convoluted as well with its corresponding frequency of a sample of an employed sub-carrier.

Such type of treatment improves the BER efficiency of convolution process due to the raised resolution of the filter to recognize the intended in-between frequencies for samples of OGFDM sub-carrier. That, however, raises the computational complexity due to an increased number of multiplications of the filtration process.

On the other hand, the proposed approach which raises the oversampling $(O V=2 K)$, can handle this problem utilising advantages of the enlarged $\alpha$ and benefit from the high flexibility in expansion between any two adjacent filters. Thus, the considerable relation between the doubled $O V$ and the expanded value of $\alpha$ factor at accepted limits of errors results in mitigating the variance among the filter coefficients. Hence, whenever the assigned value of $\alpha$ is increased, a passband ripple of the configured filter is decreased and vice-versa. 
As a result, the increased degrees of the $\alpha$ factor $(\alpha>0)$ can address this issue without the need to promote the resolution of the utilised filter.

In contrast, with the minimum oversampling $(O V=K)$, increase the value of the $\alpha$ comes up with a side effect. That's mean, further enlarged $\alpha$ factor can improve the BER performance of the filter's passband ripples but cause in an extra rise of interference between applied filters.

\section{Experimental Work}

To explore performance characteristics of the proposed OGFDM with ICIA system in terms of channel capacity and BER, a numerical simulation is extensively undertaken in the PHY of an electrical, digital, and wireless transmission system.

Through this paper, the utilised modulation formats for the frequency subcarriers vary from the low formats like the BPSK to the higher schemes as the 256 QAM which is proposed recently to the $5 \mathrm{G}$ technology [21, 22].

Besides, depending on the employed approach for interference manipulation, the oversampling factor is mainly assigned for two values, $(O V=K)$ for the conventional way, and $(O V=2 K)$ for the proposed method (ICIA), where $K$ refers to the number of applied sub-carriers.

Unless explicitly stated, four digital filters with their convoluted sub-carriers are considered. In addition, to simulate the generation and detection process for each filtered sub-carrier in the OGFDM system, the approach mentioned in [7] is adopted. Also, a sampling speed (DAC/ADC) equivalents to $4 \mathrm{GHz}$ is applied.

According to the trade-offs relation between aggregated channel capacity of the OGFDM system and the flexibility of filter design, the key guidelines of the optimum filter scheme are recognised. As such, the filter BW access can be assigned to different values (dynamic range) through this simulation but it is initially fixed at its optimal value, $(\alpha=0.1)$.

Moreover, the co-existence between the $\alpha$ value of the overlapped frequency, and the weakened ripples of the frequency response rises the opportunity of achieving an optimum value of $\alpha$, which, in turn, corresponds to a maximum channel transmission capacity.

To explain the key advantages and disadvantages of employing the ICIA, the overall channel capacity versus $\alpha$ filter parameter is examined for two adopted oversampling values, the normal one $(O V=4)$ that is used for the traditional processing of interference and the doubled method $(O V=8)$ which is employed for the developed scheme of interference manipulation (ICIA).

As is shown in Fig. 5, in comparative with the stable channel capacity of the doubled oversampling case (DOV), the fluctuations in channel capacity for normal oversampling case (NOV) are classified here into three main zones.

First is the 'Green Zone' (GZ), which represents all channel capacities above the stable line of channel capacity for the DOV. Thus, the achieved channel capacities for the NOV are better than the obtained gained DOV channel capacities. The GZ capacities are calculated for all $\alpha$ values of the period between 0 and 0.3 , where $(0<=\alpha<=0.3)$. 


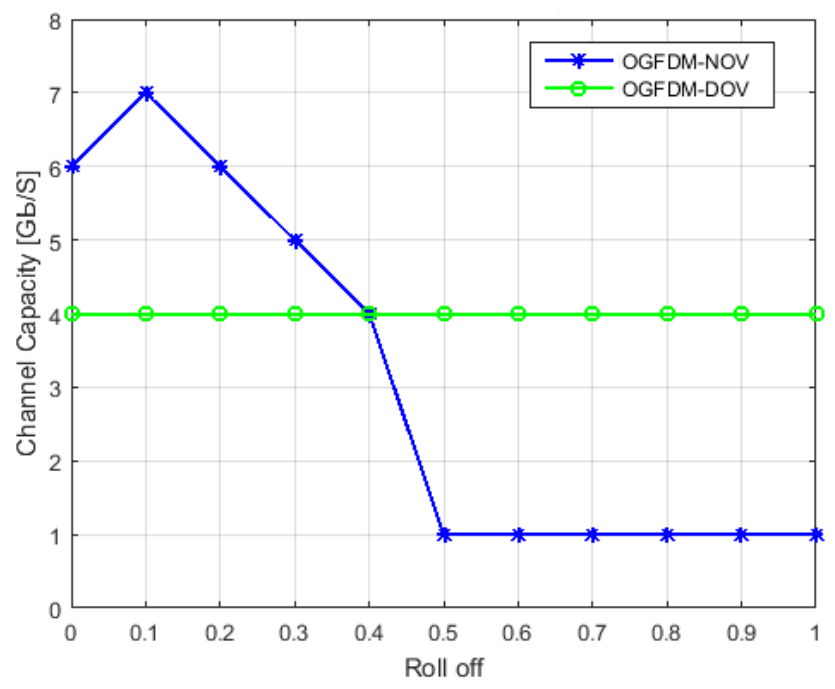

Fig. 5. Channel capacity of OGFDM with NOV/DOV for the entire range of $\alpha$

Second is the 'Yellow Zone' (YZ), which is counted for the $\alpha$ value of 0.4. In this neutral area, the channel capacities for both the NOV and DOV are equivalent. From oversampling perspective, there are two logical reigns inside the YZ (safe and alarm). Hence, employing the NOV in the YZ puts the system under the alarm reign, while utilising the DOV promotes it to the safe reign. Thus, exceed the accepted limits of the GZ and working in YZ of the NOV results in ringing the alarm system to avoid any probable increase in the $\alpha$ factor (alarm status).

Third is the 'Red Zone' (RZ), which is introduced for all channel capacities under the stable line of the DOV channel capacity. Thus, it basically refers to all $\alpha$ values between 0.5 and 1 , where $(0.5<=\alpha<=1)$. It's worth noting that the transmission system for the RZ suffers from a sharp decline in channel capacity in comparison with the utilised DOV.

For this reason, the system is converted to the alternative solution (DOV), where the decrease in channel capacities is stopped despite continuous increases in the $\alpha$ factor where $\alpha<=1$. Thus, it is necessary to replace the applied system (NOV) with the DOV to switch the context of transmission from the red to the yellow zone.

In this experiment, it is worth pointing out that using the NOV, the maximum channel capacity is achieved with the optimum value of the $\alpha(\alpha=0.1)$. In addition, depending on the best channel capacity, the ratios of other calculated channel capacities for both the NOV and DOV are rated accordingly.

For example, in the GZ, the highest proportions of channel capacity, $85.7 \%$, $85.7 \%$, and $71.4 \%$, are obtained for the $\alpha$ values of $0,0.2$ and 0.3 respectively. 
In addition, the middle ratio of channel capacity at the $\mathrm{YZ}$ is dropped to $57.1 \%$ for a $\alpha$ value equivalent to 0.4 . Moreover, the lowest ratio of channel capacity (14.4\%) is registered for the $\alpha$ values of the RZ between 0.5 and 1 .

This, however, can be improved by utilising the DOV which is able to stop the bad effect of increased $\alpha$ for values larger than 0.4 . Hence, the average ratio of channel capacity is settled at, $57.1 \%$ for all $\alpha$ values between 0 and 1 .

Nevertheless, the gained ratio of channel capacity for the $\alpha$ values between 0 and 0.3 is less than the corresponding proportions of the $\operatorname{NOV}(85.7 \%, 71.4 \%$,). Thus, the offered DOV is more suitable for the RZ than the GZ, since the ratio of obtained channel capacity is raised, in the RZ, from $14.1 \%$ to $57.1 \%$, which is equivalent to 4 times channel capacity of the NOV.

To mitigate such an issue, a hybrid solution is recommended utilising the advantages of both the NOV and DOV in one robust transmission system. In a such hybrid system, the NOV is applied for the $\alpha$ values between 0 and 0.4 while the values of $\alpha$ between 0.5 and 1 are allocated to the DOV. The hybrid treatment can produce a more robust transmission system with a minimum ratio of channel capacity equivalents to $57.1 \%$ at the worst condition and maximum ratio reaches $100 \%$ at the optimal value of the $\alpha$.

As a result, the hybrid handling can offer both the higher channel capacity and more reliability due to its strong ability in supporting the accepted levels of the $\alpha$ factor for values between 0 and 0.4 and removing the serious influence of declined $\alpha$ for values between 0.5 and 1 .

As is clear from Fig. 6, the calculated BW efficiency for the NOV has been taken firstly for the optimal value of $\alpha(\alpha=0.1)$, where maximum BW efficiencies are achieved for different points of the SNR. These various peaks of BW efficiencies with gradually reduced levels of the SNR come from increased interference among neighbouring samples at the constellation table.

On the other hand, a sharp reduction of BW efficiency is recorded for a $\alpha$ equal to 0.5 . Hence, using the NOV, the system shows a severe fluctuation in BW efficiency for different levels of the $\alpha$.

This, nevertheless, can be solved by utilising the DOV which demonstrates a steady level of BW efficiency for different values of $\alpha$, for instance, 0.1 and 0.5 . In addition, the BW efficiencies with different stages of the SNR are varied for the same induced reason of the NOV.

Despite the halved spaces of the available BW due to the dual oversampling, the rates of BW efficiency for different $\alpha$ values (best and worst) are reduced by about $40 \%$ instead of $50 \%$ due to avoiding interference between adjacent filters for similar limits of the SNR. Thus, since the obtained BW efficiency is decided by the two important factors (BW and SNR), around 10\% extra improvement is achieved by improved SNR, increasing the gained BW efficiency from $50 \%$ to about $60 \%$.

In contrast, the NOV rates of the BW efficiencies are decreased by about $85 \%$ at worst cases of the $\alpha$ parameter. The main reason beyond this is the difficulty in dealing with the exaggerated interference between adjacent filters under the bad conditions $(\alpha>=0.5)$. 


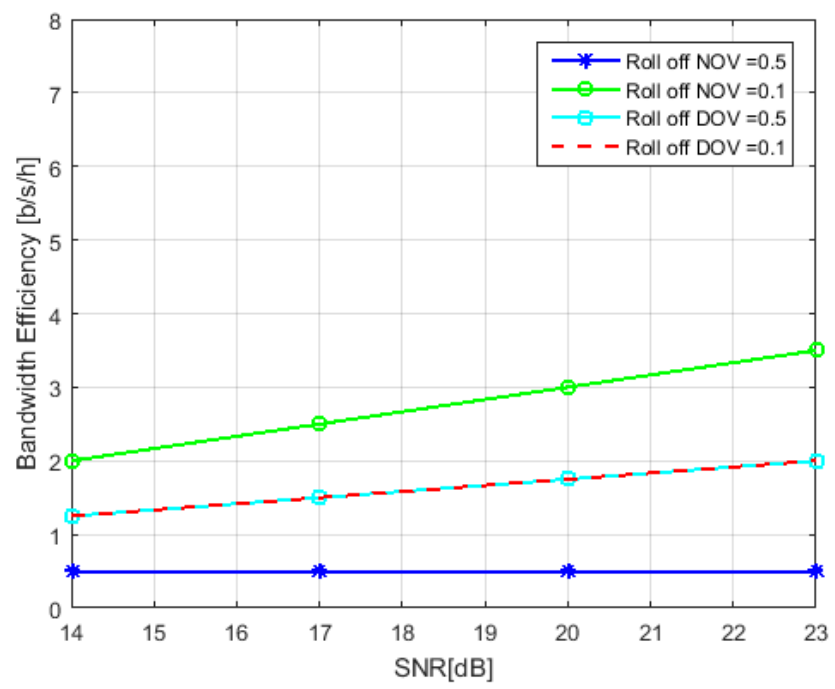

Fig. 6. BW efficiency of the OGFDM with NOV/DOV vs the SNR

It should be noted that the decreased rates of BW efficiency with both the NOV and DOV in this transmission system have been measured in comparative with the best BW efficiency of the NOV at SNR limit equivalents to 23 and $\alpha$ factor to 0.1 .

The main parameters of this experiment are shown in Table 1, in addition, they have been applied for a wireless electrical back-to-back system.

Table 1. System parameters for the OGFDM

\begin{tabular}{|c|c|}
\hline Parameter & Value \\
\hline No. of $f_{c} s$ & 2 \\
\hline$F_{D A C / A D C}$ & $4 \mathrm{GHz}$ \\
\hline SNR & 23 \\
\hline$\alpha$ & $0-1$ \\
\hline Filter length & 32 \\
\hline Spectral efficiency & $1 \mathrm{~Hz}$ \\
\hline Oversampling & Normal - Dual \\
\hline Modulation format & BPSK - 256 QAM \\
\hline OGFDM symbols & 2000 \\
\hline Filter type & Hilbert filter \\
\hline
\end{tabular}




\section{Conclusion}

The OGFDM waveform with ICIA approach has been proposed, investigated and evaluated, for the first time. The proposed system extra sustains a higher channel capacity, more data reliability and better shareable resources in the PHY than the conventional way. Since the interference manipulation is an important process for the digital filters of the OGFDM system, a thorough investigation has been undertaken to explore the interconnected relationship between the filter's roll-off factor and the level of interference. It is worth noting that with the conventional process of interference which essentially works in the NOV mode, the aggregated channel capacity is decreased more sharply with relatively increased $\alpha$ parameter $(\alpha>0.4)$. Thus, the interference between the frequency responses of the applied filters is further increased due to extremely overlapped regions between similar filter types (in-phase/quadrature-phase). On the other hand, the findings show that utilising the proposed ICIA which converts to the DOV mode, the overall channel capacity is improved, particularly, for the enlarged values of the $\alpha(0.5<=\alpha<=1)$. Hence, by adopting a doubled value of the oversampling, the bad reaction of increased $\alpha$ value is accommodated optimally. Nevertheless, in comparison with the NOV, the $\alpha$ values between 0 and 0.3 at the DOV mode result in reducing the capacity of the channel due to the narrowed BW of the filtered sub-carriers. As a result, neither the DOV nor the NOV alone can achieve the optimum interference handling. Thus, the hybrid solution that employs both (NOV \& DOV) is always recommended to boost the capacity of transmission for a diverse range of the $\alpha$ factor. Hence, such interference management presents a further optimization for the filtration process in the practical domain.

\section{Acknowledgment}

This research was funded by the Ministry of Higher Education and Scientific Research, Republic of Iraq, Scholarship 2633.

\section{References}

1. Dias, J. T. \& Lamare, R. C. De.: Unique-Word GFDM Transmission Systems. 6, 746749 (2017).

2. Yazar, A., Onat, F. A. \& Arslan, H.: New Generation Waveform Approaches for 5G and Beyond. (2016).

3. Mattera, D., Tanda, M. \& Bellanger, M.: Frequency domain CFO compensation for FBMC systems. Signal Processing 114, 183197 (2015).

4. Geng, S., Xiong, X. \& Cheng, L.: UFMC System Performance Analysis for Discrete Narrow-band Private Networks. 2015 IEEE 6th Int. Symp. Microwave, Antenna, Propagation, EMC Technol. 303307 (2015). doi:10.1109/MAPE.2015.7510319

5. Abdoli, J., Jia, M. \& Ma, J.: Filtered OFDM: A New Waveform for Future Wireless Systems. 2015 IEEE 16th Int. Work. Signal Process. Adv. Wirel. Commun. 6670 (2015). doi:10.1109/SPAWC.2015.7227001 
6. Farhang, A., Marchetti, N., Member, S., Doyle, L. E. \& Member, S.: LowComplexity Modem Design for GFDM. IEEE Trans. Signal Process. 64, 15071518 (2016).

7. Kadhum, M. R., Kanakis, T., Al-sherbaz, A. \& Crockett, R.: Digital Chunk Processing with Orthogonal GFDM Doubles Wireless Channel Capacity. 16 (2018). doi:https://doi.org/10.1007/978-3-030-01177-2-53

8. Gaspar, I. et al.: LTE-compatible 5G PHY based on Generalized Frequency Division Multiplexing. 2014 11th Int. Symp. Wirel. Commun. Syst. 209213 doi:10.1109/ISWCS.2014.6933348

9. Prabu, R. T., Benisha, M., Bai, V. T. \& Yokesh, V.: Millimeter Wave for 5G Mobile Communication Application. 2016 2nd Int. Conf. Adv. Electr. Electron. Information, Commun. Bio-Informatics 236240 (2016). doi:10.1109/AEEICB.2016.7538280

10. Chiumento, A., Pollin, S., Desset, C., Van Der Perre, L. \& Lauwereins, R.: Scalable LTE interference mitigation solution for HetNet deployment. 2014 IEEE Wirel. Commun. Netw. Conf. Work. WCNCW 20144651 (2014). doi:10.1109/WCNCW.2014.6934859

11. Kadhum, M. R.: New Multi-Carrier Candidate Waveform For the 5G Physical Layer of Wireless Mobile Networks. Proceedings of IEEE 2019 Wireless Days, Manchester, (2019).

12. Gao, W.: Energy and Bandwidth-Efficient Wireless Transmission. (2017). doi:10.1007/978-3-319-44222-8

13. Prommee, P., Thongdit, P. \& Angkeaw, K.: Log-domain high-order low-pass and band-pass filters. AEUE - Int. J. Electron. Commun. 79, 234242 (2017).

14. Widmann, A., Schrger, E. \& Maess, B.: Digital filter design for electrophysiological data - a practical approach. J. Neurosci. Methods 250, 3446 (2015).

15. Tao, L. et al.: Experimental demonstration of $10 \mathrm{~Gb} / \mathrm{s}$ multi-level carrier-less amplitude and phase modulation for short range optical communication systems. Opt. Express 21, 6459 (2013).

16. Im, G. H. et al.: $51.84 \mathrm{Mb} / \mathrm{s}$ 16-CAP ATM LAN Standard. IEEE J. Sel. Areas Commun. 13, 620632 (1995).

17. Kawamura, T. Kishiyama, Y. Higuchi, K. Sawahashi M., and Engineering, C.: Investigations on Optimum Roll-off Factor. no. 1, (2006).

18. Mukumoto, K. \& Wada, T.: Realization of Root Raised Cosine Roll-Off Filters Using a Recursive FIR Filter Structure. IEEE Trans. Commun. 62, 24562464 (2014).

19. Wang, Z., Zhou, S., Giannakis, G. B., Berger, C. R. \& Huang, J.: FrequencyDomain Oversampling for Zero-Padded OFDM in Underwater Acoustic Communications. IEEE J. Ocean. Eng. 37, 1424 (2012).

20. Yli-Kaakinen, J. et al.: Efficient fast-convolution-based waveform processing for 5G physical layer. IEEE J. Sel. Areas Commun. 35, 13091326 (2017).

21. Kadhum, M. R., Kanakis, T. \& Crockett, R.: Dynamic Bit Loading with the OGFDM Waveform Maximises Bit-Rate of Future Mobile Communications. Proceedings of Computing Conference 2019, London, (2019).

22. Haboobi, H., Kadhum, M. R. \& Al-sherbaz, A.: Utilize Higher Modulation Formats with Heterogeneous Mobile Networks Increases Wireless Channel Transmission. Proceedings of Computing Conference 2019, London, (2019). 